\title{
Ionic Interactions in Biological and Physical Systems: a Variational Treatment
}

\author{
Bob Eisenberg \\ June 7, 2012
Department of Molecular Biophysics and Physiology, Rush University, 1750 West Harrison Street, Chicago IL 60612, USA.
Fax: +312 9428711 ;
Tel: +312942 6467;
E-mail:beisenbe@rush.edu \\ As prepared for \\ Faraday Discussion on \\ Ion Specific Hofmeister Effects \\ Pavel Jungwirth Organizer \\ 3 - 5 Sept. 2012, Queens College Oxford, UK
}




\begin{abstract}
Chemistry is about chemical reactions. Chemistry is about electrons changing their configurations as atoms and molecules react. Chemistry has for more than a century studied reactions as if they occurred in ideal conditions of infinitely dilute solutions. But most reactions occur in salt solutions that are not ideal. In those solutions everything (charged) interacts with everything else (charged) through the electric field, which is short and long range extending to the boundaries of the system. Mathematics has recently been developed to deal with interacting systems of this sort. The variational theory of complex fluids has spawned the theory of liquid crystals (or vice versa). In my view, ionic solutions should be viewed as complex fluids, particularly in the biological and engineering context. In both biology and electrochemistry ionic solutions are mixtures that are highly concentrated $(\sim 10 \mathrm{M})$ where they are most important, near electrodes, nucleic acids, proteins, active sites of enzymes, and ionic channels. $\mathrm{Ca}^{2+}$ is always involved in biological solutions because the concentration (really free energy per mole) of $\mathrm{Ca}^{2+}$ in a particular location is the signal that controls many biological functions. Such interacting systems are not simple fluids, and it is no wonder that analysis of interactions, such as the Hofmeister series, rooted in that tradition has not succeeded as one would hope. Here, we present a variational treatment of hard spheres in a frictional dielectric with the hope that such a treatment of an electrolyte as a complex fluid will be productive. The theory automatically extends to spatially nonuniform boundary conditions and the nonequilibrium systems and flows they produce. The theory is unavoidably self-consistent since differential equations are derived (not assumed) from models of (Helmholtz free) energy and dissipation of the electrolyte. The origin of the Hofmeister series is (in my view) an inverse problem that becomes well posed when enough data from disjoint experimental traditions are interpreted with a self-consistent theory.
\end{abstract}




\section{Introduction}

Science flows in certain directions because scientists, like all people, are creatures of our training. Theories taught in our youth, before we learn critical skills, are hard for us to see objectively, and even harder to modify. But modify we must, as disciplines overlap, questions intermingle, and methods evolve. Modify we must if important questions remain unsolved despite the diligent work of generations of scientists.

\section{Chemistry}

Chemistry is first of all about chemical structures and reactions on the atomic scale. Chemists were excited by molecules and flows of electrons within those structures. Chemistry evolved without much thinking about macroscopic flow of molecules in space and time. Chemistry made remarkable advances because of this tight focus on chemical reactions. Physical chemists studied the tiny part of phase space that describes systems without macroscopic flow. The immense power of equilibrium thermodynamics and statistical mechanics $^{1-3}$ culminated in the theory of simple liquids, with its cathedrals ${ }^{4-8}$ of analysis. The theory of simple liquids provides elegant insights of power and complexity. The founders of thermodynamics would surely have shared my view that the theory of simple liquids is an aesthetic and scientific masterpiece.

\section{Simulations}

In the last decades, aesthetics have often been replaced by calculations. Simulations have replaced much of the theory of simple liquids, judging by the number of papers published, but simulations have not yet dealt successfully with most macroscopic phenomena in my opinion ${ }^{9}$, because in most cases simulations have not yet been able to confront macroscopic phenomena in the form found in the laboratory ${ }^{10-47}$ and in chemical engineering every day. $^{48}$

As the simulation profession adopts the standards of validation of the experimental community $^{48-50}$, it may well replace analysis as the best connector between atomic scale descriptions of chemical reactions and macroscopic measurements of the output of these reactions. But that has not happened yet, in my view and the consequences are serious.

Simulations are not mathematics, as much as I wish they were. Simulations cannot rely on the theorems that buttress and underlie numerical analysis. In my view, simulations need to be continually checked by comparison with test cases (e.g., conservation laws, model systems with known properties) just as do experiments. Until they fit experiments in conditions similar to those of interest, they have limited use. In the case of biological systems, those conditions are troublesome. All of life occurs in salt solutions derived from oceans. Without the proper ionic environment, cells burst, proteins coagulate, life stops. If simulations are not performed in solutions resembling those of life, they clearly cannot fit the properties of those solutions. Thus, simulations must be done in mixtures of $\mathrm{Na}^{+}, \mathrm{K}^{+}, \mathrm{Ca}^{2+}$ and $\mathrm{Cl}^{-}$ions of substantial concentration $(\sim 0.5 \mathrm{M})$. Biological solutions are mixtures always involving $\mathrm{Ca}^{2+}$ ions over a wide range of concentrations $\left(10^{-8} \mathrm{M}\right.$ as intracellular signals; $\sim 10^{1}$ $\mathrm{M}$ near and in active sites, nucleic acids, and ion channels). I am unaware of simulations done in these conditions, although surely some will be done soon enough. Until simulations reproduce the properties of life's solutions that are essential to maintain life, they are in a never-never land, in which their awesome detail and power can actually mislead. As any experimental biologist knows, uncalibrated measurements, or solutions of unknown 
composition can produce a bewildering array of results, that take more than a lifetime to sort out, most of which are artifacts, having little to do with biological function. One cannot expect uncalibrated simulations to do better than uncalibrated experiments.

\section{Mathematics}

Applied mathematics has a history quite different from chemistry. Applied math has always described macroscopic movement and flow. Indeed, mathematics of flow developed rapidly in the 1800's before molecules were thought to be real by many physicists.

Mathematics developed so powerfully that measurements of infinitesimal changes in fluids could be transformed into successful predictions of macroscopic flows. Infinitesimal measurements could be describe by infinitesimal (partial differential) equations (e.g., Navier Stokes) describing matter within boundaries. The meaning of the symbols in the equations could be forgotten, and the differential equations treated as abstractions. The abstractions could be integrated analytically and numerically (as mathematical objects without additional physical assumption).

An infinitesimal treatment could tell us what large masses of fluids do after the infinitesimals were integrated into macroscopic reality by mathematicians who knew nothing of what they were integrating. ${ }^{51,52}$ Water flowing in showers, water waves in oceans, even the action of wind on the water could be determined by mathematics. Indeed, computational fluid dynamics can predict complex flows (with shock waves, over complex structures) like those of air around a supersonic airplane, with a power and accuracy that leaves outsiders like me breathless.

\section{Mathematics is Different}

Mathematics learned to deal with certain classes of systems (conservative systems without dissipation) in a more abstract way, using the variational treatment of Hamilton. This variational approach had the important advantage of dealing with interactions automatically by algebra. The differential equations of motion were derived from the Least Action Principle. Components could be added to the Hamiltonian and the interactions of the components and the differential equations of motions re-derived for the composite system. The interactions of the new and old components appeared automatically in the differential equations without further physical argument. In this way, complex systems could be studied without ad hoc physical study of interactions, if the original and composite systems involved the same physics described by similar Hamiltonians.

The complex systems of the mathematicians did not involve chemicals, sad to say. The chemicals of the fluids and the atoms of the Hamiltonian were ignored by mathematicians, just as the interactions and macroscopic flow of the chemicals were ignored by the chemists, for many years. Chemicals were mysterious shadows to most mathematicians, as partial differential equations were mysterious moving shadows, a cinema of darkness, to most chemists.

\section{An interdisciplinary attempt to fill in the shadows}

I argue here that problems like the physics and physical chemistry of the Hofmeister series should be viewed in a new light, the additional light of mathematics, with a good measure of engineering insight thrown in. The light of just one discipline casts shadows too deep to see into. Light from another direction can fill those shadows substantially. Of course, the light of the other discipline creates its own shadows. So the trick is to use each 
discipline's light and ignore its shadows, melding this vision into a coherent self-consistent view of the entire phenomena. It should go without saying that I do not know how to do that, but I do think I know where to aim the light.

The essential difficulty in dealing with Hofmeister effects is, as I see it, that everything depends on interactions. To put it crudely, in the ideal noninteracting world, there is no Hofmeister series.

\section{Inverse Problems: reverse engineering the Hofmeister Series}

Of course, saying everything depends on interactions says too much, and says it too vaguely. There are many types of interactions and keeping them separate is essential if we are to avoid confusion. We need to keep the interactions if we wish to calculate what a solution would do from first principles.

The problem is much worse if we face it backwards, as I think we actually do. We are actually trying to infer the atomic basis of the Hofmeister series from macroscopic data taken under a wide range of conditions. This is an inverse problem, a problem in reverse engineering. Such problems are notoriously tricky because they are both ill-posed and overdetermined, in the typical case. Sorting out the class of physical interactions is crucial if we are to make progress with our inverse problem. ${ }^{53,54}$ As difficult as the problems seem, there is real hope of progress, at least in the biological case, when the natural selection of evolution has created a reasonably robust device described by reduced equations, ${ }^{55}$ as it apparently has when dealing with the selectivity of calcium ${ }^{56,57}$, sodium $^{58}$, and ryanodine receptor ${ }^{59}$ channels.

Let's start with the simplest case. Let's talk about an implicit solvent model of ionic solutions, in which ions are hard spheres, of different charge and diameter, in a dielectric which also provides friction for flow. We consider the possibility that the Hofmeister effect might arise in even this oversimplified system ${ }^{60}$ because of interactions that have not been dealt with self-consistently by previous methods. Of course, it is much more likely (from the chemists' perspective) that additional physics, beyond that of the implicit solvent primitive model will be needed to produce the effect. From the mathematicians' perspective, one cannot tell what a model is unless it is computed self-consistently, so the mathematician has no opinion of earlier work.

Consider a homogeneous system of say $\mathrm{NaCl}$. We have spheres of two diameters and two charges. Monte Carlo simulations of this system do surprisingly well compared to real experimental data ( $\mathrm{see}^{18}$ for a recent treatment and references to the enormous literature). Monte Carlo simulations also do well for another monovalent salt say $\mathrm{KCl}$. If we mix $\mathrm{NaCl}$ and $\mathrm{KCl}$ over a range of concentrations, difficulties arise because of interactions of the hard spheres within the physical model I just described. If divalents are involved $-\mathrm{CaCl}_{2}$, for example-we start with pure simulations of pure $\mathrm{CaCl}_{2}$, and then mix $\mathrm{CaCl}_{2}$ with $\mathrm{NaCl}$ and/or $\mathrm{KCl}$.

Mixed solutions have many interaction terms. Even if the physics in these mixtures is the same as in the pure solution (spatially uniform dielectric coefficient, etc.), the interactions are difficult to deal with. So many terms are involved, and theories and measurements have enough uncertainties, that it is very difficult to evaluate the coefficients of interactions.

In fact, mixed solutions of this type are very important to chemical engineers and have received a great deal of attention by them, summarized in ${ }^{40,43,61}$. Nonetheless, it seems clear that the many attempts to create a coherent self-consistent model of the activity (free 
energy per mole) of ionic mixtures has been a difficult one. A generation of physical chemists $\operatorname{tried}^{38,42,43,45-47,62-69}$ culminating in the compendium of reference ${ }^{43}$ that summarized the work of a taskforce of chemical engineers funded for many years for this purpose. Equations of state were awkwardly complex (summarized in $^{70}$, see also ${ }^{71-78}$ ). Apparently promising simplifications ${ }^{40,69,79}$ seem not to have taken hold. Modern treatments and compendia of results for chemical engineers ${ }^{29,} 61$ seem to lack physical basis or 'transferability'. That is to say, models and parameters developed for one type of mixture do not apply to other mixtures and other cases.

Enormous efforts of many workers (only some of which I know about ${ }^{10-27,30,32-34,59,}$ 80-108) have not produced a coherent view of ionic mixtures, containing divalents, as far as I can tell. This is not just a biologist's view, looking from the outside. A recent volume ${ }^{29}$ described progress in dealing with specific properties of ionic solutions of many groups. An overview article by Kunz and Neuderer ${ }^{28}$ concluded

"It is still a fact that over the last decades, it was easier to fly to the moon than to describe the free energy of even the simplest salt solutions beyond a concentration of $0.1 \mathrm{M}$ or so."

This situation is rather upsetting, particularly to me as a biologist, mostly interested in biological solutions, because our solutions are those that Kunz and Neuderer say are not well described, let alone understood.

Biology occurs in salt solutions evolved from the oceans of the world. Biological salt solutions are invariably mixtures of $\mathrm{NaCl}$ and $\mathrm{KCl}$ with $\mathrm{CaCl}_{2} \cdot \mathrm{Ca}^{2+}$ plays a particularly important role because the concentration of $\mathrm{Ca}^{2+}$ acts as a specific control signal for many of life's processes. Indeed, $\mathrm{Ca}^{2+}$ signals are as important and diverse in biology as electrical voltage is in computers! The quotation of Kunz and Neuderer implied what I feared ${ }^{109-111}$ : the physical chemistry of life's solutions is not understood.

Here I suggest a different approach. I present a new variational treatment of ionic solutions ${ }^{112-115}$ that produces (nearly) unique results for mixtures. I propose that this treatment can be used to systematically refine treatments of mixtures allowing a (hopefully) rapid convergence to a full understanding. But this is an approach not a result. The computations to compare with specific experiments have not been done. The feasibility of those computations has been shown, but the detail necessary to compare with results has not been computed.

\section{The variational approach}

The approach is to start with a simple model of a pure solution (say the primitive model of $\mathrm{NaCl}$ ). Write it in a variational form. Derive the appropriate differential equations by the Euler Lagrange process. Learn to integrate those equations efficiently in a setup that allows evaluation of activities. Compare with experiment. definite.

The approach repeats the same procedure with another pure solution, say $\mathrm{CaCl}_{2}$ to be

Then a variational model of a mixture of $\mathrm{NaCl}$ and $\mathrm{CaCl}_{2}$ is made (by combining energy and dissipation) and converted into differential equations with boundary conditions appropriate for the experimental setup. The resulting differential equation has minimal adjustable parameters. Most coupling terms are determined by the Euler Lagrange process itself. The differential equation for the mixture is then integrated and applied in conditions 
that determine activity coefficients of the components of the mixture. Comparison is made with experiments.

When conditions are found in which the model fails to fit experiments, additional physics is included in the variational model. For example, a specific model of electron interaction between $\mathrm{Ca}^{2+}$ and water is made, or a specific model of the spatial variation of dielectric coefficient is made, and included in the variational model. This model is then converted into a set of differential equations for mixtures just as was the primitive model, in the last few paragraphs.

\section{Ionic Solutions as Complex Fluids}

Ionic solutions are complex fluids because they contain charged particles. Ions in solution interact so strongly through the electric field that they always come 'in pairs' (within $\sim 1$ part in $10^{15}$ ). Ionic solutions are necessarily neutral or they explode. ${ }^{116}$ Most ions also interact through their finite size. ${ }^{23,29,35,62,63,111}$ At biological concentrations, the shape of the ion dramatically distorts the shape of the electric field. The electric field of $1 \mathrm{M}$ spheres (approximately $8 \AA$ apart) of diameter $2 \AA$ is not well approximated by the electric field of $1 \mathrm{M}$ points. Finite size ions cannot occupy the same space, so ionic solutions are particularly complex in crowded confines near electrodes, active sites, and ion channels. Devices that use ions often depend on the nonideal properties of crowded ions. Ionic solutions are not simple fluids, particularly where they are most important, i.e. where they are crowded. Ionic solutions need to be treated as the complex fluids that they are, in my opinion.

It is best now to add some specifics to these abstractions. To do that, we consider a specific variational model of ionic solutions, ${ }^{112-115,117}$ that has grown from the theory of complex fluids ${ }^{118-126}$ developed in the context of liquid crystals, ${ }^{120,122,127-129}$ more than anything else.

The approach uses the dissipation principle as described by $\mathrm{Liu}^{118}$ and co-workers built on the analysis of dissipative systems of Rayleigh [Strutt], ${ }^{130-132}$ Onsager, ${ }^{133-136}$ and Doi, explicitly $^{121,137}$ and implicitly, ${ }^{129}$ among many others ${ }^{11}$

We start with the key fact that the ions in water satisfy the dissipation law

$$
\begin{aligned}
\frac{d}{d t} \int \overbrace{\left\{k_{B} T \sum_{i=1}^{N} c_{i} \log c_{i}+\frac{1}{2}\left(\rho_{0}+\sum_{i=1}^{N} z_{i} e c_{i}\right) \phi\right\}}^{\text {Helmholtz Free Energy Conservative Term }} d \vec{x} \\
=-\underbrace{-\int\left\{\sum_{i=1}^{N} \frac{D_{i} c_{i}}{k_{B} T}\left|k_{B} T \frac{\nabla c_{i}}{c_{i}}+z_{i} q \nabla \phi\right|^{2}\right\} d \vec{x}}_{\text {Raleigh [Strutt] Dissipation Term }}
\end{aligned}
$$

This ensures that ions follow both an Energetic Variational Principle and also a set of simply derived differential equations. Either can be derived from the other. 


$$
\frac{d E}{d t}=-\Delta
$$

or

$$
\frac{\delta E}{\delta \vec{x}}=\frac{1}{2} \frac{\delta \Delta}{\delta \vec{u}}
$$

or

$$
\underbrace{\text { Conservative Force }}_{\begin{array}{c}
\text { from variation with respect to } \\
\text { Position } \vec{x}
\end{array}}=\underbrace{\text { Dissipative Force }}_{\begin{array}{c}
\text { Drom variation with respect to } \\
\text { Velocity } \vec{u}
\end{array}}
$$

$E$ is the Helmholtz free energy; $\Delta$ is the Rayleigh[Strutt]/Onsager dissipation discussed at length in the literature. ${ }^{12,118,121,130-136,138,139}$

I think it better physically to start with the variational principle because additional fields or components are best handled that way. The variational form of this expression allows easy derivation of the Euler Lagrange differential equations, which can also be written down directly in this case.

$$
\begin{gathered}
\frac{\partial c_{i}}{\partial t}=\nabla \cdot\left(D_{i}\left(\nabla c_{i}+\frac{z_{i} e}{k_{B} T} c_{i} \nabla \phi\right)\right) \\
\nabla \cdot(\varepsilon \nabla \phi)=-\left(\rho_{0}+\sum_{i=1}^{N} z_{i} e c_{i}\right) .
\end{gathered}
$$

These are the usual Poisson Nernst Planck equations, called the Vlasov equations in plasma physics ${ }^{143-145}$, drift diffusion in semiconductor physics ${ }^{146-151}$ and PNP first ${ }^{152}$ in biophysics $^{11,55,106,111,117,153-212}$ and then in electrochemistry ${ }^{158,200,213-215}$ and applied mathematics, ${ }^{11}, 106,117,146,148,149,158,159,165,186,202-204,206-209,213,214,216$ in which $c_{i}$ are the number densities of ions of species $i$, with diffusion coefficient $D_{i}$, valence $z_{i}$, charge $z_{i} e$ with $e$ the unit charge of a proton, diffusion (free) energy of $k_{B} T$, at electrical potential $\phi$, in permittivity $\varepsilon$, with protein permanent charge density $\rho_{0}$ as it represents the charge density of doping in semiconductors. $\rho_{0}$ depends on location and is zero in bulk solutions.

The coupling of these equations is tricky because the net charge $\sum_{i=1}^{N} z_{i} e c_{i}$ is well determined by the Laplacian of the electrical potential but cannot (in essence) be computed by summing the individual concentrations $c_{i}$ because the charge weighted sum is so tiny $\left(\sim 10^{-15}\right)$ compared to individual concentrations. The system must be (nearly) electroneutral or potentials would strip electrons from atoms.

This treatment so far deals with point ions making a solution that is nonideal in a simple way. Interactions are only electrostatic, as in classical Debye-Hückel and Poisson Boltzmann equilibrium theories. This is not the primitive model. 


\section{Spatially Nonuniform Boundary Conditions}

The variational method and the resulting Poisson Nernst Planck (PNP)11, 55, 106, 109, 117, 146-148, 151-153, 155, 159, 163, 164, 169, 170, 172-175, 179, 183, 184, 188, 190, 193, 194, 197, 201, 202, 204, 206, 207, 209-212, 214-219 equations have substantial advantages, however, over the traditional equilibrium approach. The PNP equations allow spatially nonuniform boundary conditions such as those needed to drive flux. In our electronic technology those spatially nonuniform boundary conditions are the power supply potentials. In biology, those spatially nonuniform boundary conditions are the electrical potential and concentration gradients across the cell membrane. In chemistry, those spatially nonuniform boundary conditions are the concentrations of the classical Nernst equation of electrochemistry and polarography, and perhaps an applied electrical potential as well.

A great deal is gained by using PNP as a nonideal theory allowing flow: all of the components of a computer can be built using circuit elements that satisfy PNP.148-151, 220231 Resistors, capacitors, and transistor switches, transistor amplifiers, etc. are actually mathematical solutions of the PNP equations. Thus, moving to PNP allows one to write "all human knowledge and logic" (that can be performed by a computer) as a solution of a partial differential equation in a spatially complex domain with spatially nonuniform (but otherwise simple) boundary conditions.

\section{Finite Size of Ions}

To deal with the finite size of ions, and extend PNP into a theory of ionic solutions, we need to decide how to describe the finite diameter of the ions. This decision is surely not unique, ${ }^{10-12,15,16,19,22-25,27,100,103,104,106,232}$ and the choice of description depends on the feasibility of calculations and the accuracy of fit of the resulting model to a broad range of data, from different types of experiments, in a broad range of solutions and concentrations. This work has not yet been done. The purpose of this paper is to facilitate and motivate such work.

We have included finite size at the length scale of PNP by adding terms to the above equations. The resulting model is variational, so it is a model in the spirit of the theory of complex fluids, but the fluid is the primitive model of electrolytes, leading to peculiar oxymoronic names for our model, like 'complex primitive theory' of electrolytes! Given the present state of checking of our models, (oxy)moronic names are perhaps appropriate.

To be specific, our variational theory describes the (Helmholtz free) energy and dissipation of ionic solutions by

$$
\begin{aligned}
\frac{d}{d t} \overbrace{\left\{k_{B} T \sum_{i=n, p} c_{i} \log c_{i}+\frac{1}{2}\left(\rho_{0}+\sum_{i=n, p} z_{i} e c_{i}\right) \phi+\sum_{i, j=n, p} \frac{c_{i}}{2} \int \tilde{\Psi} c_{j} d \vec{y}\right\} d \vec{x}}^{\text {Conservative }} \\
=-\underbrace{\int\left\{\sum_{i=n, p} \frac{D_{i} c_{i}}{k_{B} T}\left|k_{B} T \frac{\nabla c_{i}}{c_{i}}+z_{i} e \nabla \phi-\sum_{j=n, p} \nabla \int \tilde{\Psi}_{i, j} c_{j} d \vec{y}\right|^{2}\right\} d \vec{x}}_{\text {Dissipative }}
\end{aligned}
$$


$\underset{i, j}{\Psi}$ represents the Lennard Jones crowded charges terms defined in references ${ }^{112,118}$. The variational formulation and variational derivatives are computed and discussed in those papers.

If ions are modeled as Lennard Jones spheres, the variational principle produces 'Euler Lagrange' equations of a drift-diffusion theory with finite sized solutes that are a generalization and correction of PNP.

$$
\begin{aligned}
\frac{\partial c_{n}}{\partial t}=\nabla \cdot\left[D _ { n } \left\{\nabla c_{n}+\frac{c_{n}}{k_{B} T}\left(z_{n} e \nabla \phi-\int \frac{12 \varepsilon_{n, n}\left(a_{n}+a_{n}\right)^{12}(\vec{x}-\vec{y})}{|\vec{x}-\vec{y}|^{14}} c_{n}(\vec{y}) d \vec{y}\right.\right.\right. \\
\left.\left.\left.-\int \frac{6 \varepsilon_{n, p}\left(a_{n}+a_{p}\right)^{12}(\vec{x}-\vec{y})}{|\vec{x}-\vec{y}|^{14}} c_{p}(\vec{y}) d \vec{y}\right)\right\}\right]
\end{aligned}
$$

always combined with the Poisson Equation

$$
\nabla \cdot(\varepsilon \nabla \phi)=-\left(\rho_{0}+\sum_{i=1}^{N} z_{i} e c_{i}\right) \quad i=n \text { or } p
$$

We write the equation only for negative monovalent ions with valence $z_{n}=-1$ to keep the formulas reasonably compact. Programs have been written for all valences. $c_{p, n}(\vec{y})$ is the number density of positive $p$ or negative $n$ ions at location $\vec{y} . \varepsilon_{n, n}$ and $\varepsilon_{n, p}$ are coupling coefficients. $a_{p_{\text {or } n}}$ are the radii of ions.

\section{Field Theory of Ionic Solutions.}

These equations form a field theory of electrolytic solutions and so pretend to explain all phenomena of those solutions, at equilibrium and in flow. They automatically are selfconsistent and change form as new ions are added into the system. Thus, these equations form a necessarily self-consistent model of the flow of mixtures of ionic solutions. Up to now, most such models have been rather ad hoc, and one could not be sure if they were selfconsistent or not. ${ }^{44,233-253}$ Additional fields ${ }^{254}$ (e.g., heat flow ${ }^{138}$ or convection $^{12,}{ }^{115}$ ) can be added to our variational model by including their (Helmholtz free) energy in the energy functional $E$ and the dissipation in the dissipation functional $\Delta$ of equation (2).

Equations of this generality can be misleading, however, because difficulties often appear in the numerical implementation. Work to date shows that the calculations needed to describe electrochemical cells and ion channel are feasible and not particularly irksome, but it took some years to reach that conclusion. Each experimental setup requires translation into an approximate model, and then numerical procedures must be designed that focus work on the important features of the model these difficulties can be overcome one by one, as they have been in other models of the experimental setups.

\section{Applications to Specific Problems}

The field theory of ionic solutions has been applied to a number of problems of biochemical and chemical interest. The original publications ${ }^{12,113}$ computed curves of binding selectivity in two classical channels of considerable biological interest the calcium channel of cardiac muscle ${ }^{57,255-268}$ and the voltage activated sodium channel of nerve. ${ }^{269-277}$ 
Both were represented by the implicit solvent model with side chains represented as spheres free to move within the channel selectivity filter, but not able to move out of that region. ${ }^{56-58}$, 111, 267, 278, 279 This model has proven remarkably successful in dealing with the important selectivity phenomena of these channels. A single model, with one set of parameters that are never changed, using crystal radii of ions, and one dielectric coefficient and one channel diameter, is able to account for selectivity data in a wide range of solutions (over 4 orders of magnitude of calcium concentration, and in solutions of varying $\mathrm{K}^{+}, \mathrm{Na}^{+}, \mathrm{Rb}^{+}$and $\mathrm{Cs}^{+}$ concentration, for example). The calcium channel is represented by side chains Glu Glu Glu Glu and the sodium channel by Asp Glu Lys Ala. This work is reported in some 35 papers using various numerical methods including the variational model described here. It has been possible to invert the procedure. The inverse problem of determining the distribution of side chains inside the channel from current voltage relations in a range of solutions has actually been explicitly solved, using established methods of inverse problems, including the effects of noise and systematic error. ${ }^{55}$

The variational method allows calculations of current vs. time and current vs. voltage not previously performed on this reduced model of calcium and sodium channels. Such calculations are of some significance since they confront the measurements performed by hundreds of membrane biologists following the paradigms introduced by Hodgkin and Huxley, reviewed in reference ${ }^{280}$.

The variational method was extended ${ }^{115}$ to allow computation of the combined effects of water flow and electrodiffusion that underlie the function of many tissues and cells, from kidney, to epithelia, to cerebrospinal fluid and so. Indeed, classical physiology from Harvey to Pappenheimer ${ }^{281,282}$ might be considered the study of the flow of water and ions. As far as we know, this paper represents the first selfconsistent treatment of this classical subject, allowing cell volume changes, convection, and ionic electrodiffusion.

The application of the variational method to classical problems of physical chemistry has started with the classical charged wall problem. There we have shown ${ }^{283}$ direct comparisons with the Metropolis Monte Carlo calculations of Henderson and Boda ${ }^{86,92,284-}$ 289 , which form a significant fraction of our understanding of ionic solutions in homogeneous and inhomogeneous situations.

The most striking success of these reduced models has been the description of the main calcium channel that controls contraction in muscle (and appears in neurons and many other cell types as well, so far with unknown function), the Ryanodine Recptor Ryr. ${ }^{290-297}$ Gillespie has shown that an extension of the reduced model called PNP-DFT ${ }^{185,298,299}$ does remarkably well ${ }^{59,268,300-303}$ in predicting experiments of some complexity and subtlety (i.e., anomalous mole fraction effects and three cation mixtures) as well as drastic mutations changing charge densities from some 13 molar to zero. The theory used was developed ${ }^{185,297,}$ 298 before selfconsistent variational methods were known to me, and is not selfconsistent. It leaves out the effects of changes in shape of the ionic atmosphere thought to be important 'forever', 44, 235-240, 247, 249, 304 and now found in textbooks. ${ }^{35,36,305}$ These effects may in fact be unimportant in applications to channels, where the shape of the ionic atmosphere may be mostly determined by a (rather) unchanging protein. But the lack of self-consistency in PNPDFT prevents it from being a good model of ionic solutions in physical chemistry, where changes in shape of the ionic atmosphere are thought to be dominant. ${ }^{44}$ The variational treatment of ionic conductance ${ }^{112,113,115,118,283}$ makes no simplifying assumptions about the 
shape of the ionic atmosphere: everything (that is in the model) interacts with everything else, to minimize the dissipation and (Helmholtz free energy) of the model.

This work is more practical science than vague theory. It is meant to connect the mathematical results of the theory of complex fluids with the practical problems of experimental biophysicists and physical chemists. Sadly, the authors know much less of those problems in physical chemistry than in biophysics, in which they have worked for many years. Thus, the utility of this approach for classical physical chemistry remains to be seen. Applications to the Hoffmeister series, and the experiments that define the Hoffmeister series are one important part of our approach to classical physical chemistry.

\section{Experimental Setups}

The experimental setups used to measure the Hofmeister series are varied and in my view must be understood in some detail before they can be well modelled by this new approach. The subtleties are nontrivial, as can be seen in the magnificent treatise of Hünenberger and Reif ${ }^{16}$ which describes thousands of papers using hundreds of methods (I estimate) to determine the free energy per mole of individual ions. Many different setups are used and each requires a separate idealization, model, and mathematical description. In my view, theories should be tested against idealizations of experimental setups and not against metaphorical abstractions like thermodynamic 'theorems'. Too often, the theorems of thermodynamics and statistical mechanics have hidden assumptions in my personal opinion. Fitting data to metaphors is likely to cause even more confusion in that case, compounding the felony, as the legal saying goes. Fitting to real data avoids this problem, at the cost of some complexity in analysis.

Application of our variational model to the Hofmeister series requires models and calculations of the free energy per mole of individual ions. Those calculations will need the help of workers familiar with the experimental setups. As the primitive model for the Hofmeister series ${ }^{60}$ - presented here in variational clothing-fails to fit some data, as it surely will, additional physics will no doubt need to be added, and the less primitive models will then have to be compared to experiments. It is not at all clear how much improvement a fully self-consistent model will provide at equilibrium in solutions of one salt. One may expect more improvement when interactions are larger and more complex, in concentrated environments, in mixtures, and in divalents. Of course, those are just the characteristics of biological solutions, so one can expect variational models to be most important as they are applied to living systems.

Along the way, the variational method will also allow introduction of boundary conditions and nonequilibrium properties that arise from spatially nonuniform concentrations and voltages. In my view, this is an important social and scientific advantage. Socially, this unites the classical thermodynamic approach (which had been isolated in its thermodynamic limit) with the classical field theories of the rest of physics. Scientifically, the nonequilibrium systems have properties of enormous importance that provide, for example, our modern electronic technology, and also allow voltage signals ('action potentials') to propagate along our nerve fibres.

\section{Specific Plan to implement a variational approach}

I present a specific plan that might implement these ideas. I am very aware that it is dangerous and presumptuous to present any plan for science. Science proceeds best when it 
is initiated and energized by the curiosity of individuals. The following is meant to provoke discussion to test the utility of the variational approach.

1) identify a specific set of experimental measurements that operationally define 'the Hofmeister Effect'

2) make a physical model of the setup of those experiment.

3) make a model of how estimates of parameters are made from the measurements of that experiment

4) make a mathematical model of the physical model, in the spirit of the variational theory of complex fluids

5) derive the field theory (Euler Lagrange) equations of the model

6) solve the equations of the model for the experimental boundary conditions.

7) compute estimates of parameters from the mathematical solutions of the equations, treating the output of the equations as if they were outputs from the experimental setup.

8) compare with experiments in a range of solutions and concentrations.

9) revise the model to include more and different physics to see if the fit to experiments is improved.

\section{Chemical Conclusions}

Chemistry has focused on atoms and molecules with less concern about the surrounding environment, understandably enough, since mathematics was not available to deal with systems in which 'everything' interacts with 'everything' else. The solutions containing these atoms and molecules were treated as ideal fluids, in which nothing interacts with nothing, if my lapse in grammar may be excused. The variational theory of complex fluids has successfully dealt with systems in which everything interacts with everything else over many scales.

\section{Chemical Reactions in the Liquid State}

In my admittedly biased view, chemical reactions in the liquid state should be analysed in the framework of complex fluids. Chemical reactions are interactions of reactants (microelements in the language of the theory of complex fluids) involving rearrangements of the internal (electronic) structure of reactants, according to the wave equation of the electron, Schrödinger's equation. Daunting interactions of electrons, microelements, and the macroscopic world can be handled automatically and self-consistently in the theory of complex fluids that cannot easily be handled by any other method I know of.

\section{Future History of Physical Chemistry}

The future history of physical chemistry will deal with ionic solutions as complex fluids, I believe. When mixtures and divalents and flow are involved, the variational approach to complex fluids is likely to lead to substantial progress. The crucial step is to force the theory to confront the actual properties of such solutions as measured with many techniques under many conditions. Determining an appropriate model of ionic solutions is an inverse problem that requires masses of disjoint data, and a variational theory, to provide useful well-posed results. 


\section{Acknowledgement}

This paper is possible because of the support of others. Chun Liu, more than anyone else, has developed the energetic variational approach to the theory of complex fluids (and liquid crystals) and spared no effort to help me understand how we can apply it to ions in liquids and channels. The physical chemistry community in general, represented in the present instance by Pavel Jungwirth, has welcomed my view ${ }^{9,109-111,266,306}$ of long standing approaches in a most generous way, in the best of the academic tradition, with criticism and scepticism to be sure, but always listening. The computational electronics community ${ }^{195,307-}$ 317 has been similarly generous. Biophysicists have not always been like that. ${ }^{9,109-111,163,164,}$ 171, 177, 266, 306, 318-321 Support was from Rush Medical College and I am particularly grateful for the administrative tasks the Dean's Office has not asked me to do. 


\section{References}

1. L. Boltzmann, Lectures on Gas Theory, University of California, Berkely CA, 1964.

2. S. G. Brush, The Kind of Motion We Call Heat, North Holland, New York, 1986.

3. E. Garber, S. G. Brush and C. W. F. Everitt, Maxwell on Molecules and Gases, MIT Press, Cambridge MA, 1986.

4. J. Barker and D. Henderson, Reviews of Modern Physics, 1976, 48, 587-671.

5. J.-L. Barratt and J.-P. Hansen, Basic concepts for simple and complex liquids, Cambridge University Press, 2003.

6. J.-P. Hansen and I. R. McDonald, Theory of Simple Liquids, First Edition edn., Academic Press, New York, 1986.

7. J.-P. Hansen and I. R. McDonald, Theory of Simple Liquids, Third Edition edn., Academic Press, New York, 2006.

8. S. A. Rice and P. Gray, Statistical Mechanics of Simple Fluids, Interscience (Wiley), New York, 1965.

9. B. Eisenberg, The Journal of Physical Chemistry C, 2010, 114, 20719-20733.

10. S. Tazi, J. J. Molina, B. Rotenberg, P. Turq, R. Vuilleumier and M. Salanne, The Journal of chemical physics, 2012, 136, 114507-114512.

11. Q. Zheng, D. Chen and G.-W. Wei, Journal of Computational Physics, 2011, 230, 52395262.

12. T. Xiao and X. Song, The Journal of chemical physics, 2011, 135, 104104-104114.

13. M. M. Reif and P. H. Hunenberger, The Journal of chemical physics, 2011, 134, 144103144130 .

14. M. M. Reif and P. H. Hunenberger, The Journal of chemical physics, 2011, 134, 144104144125.

15. J. J. Molina, J.-F. Dufreche, M. Salanne, O. Bernard and P. Turq, The Journal of chemical physics, 2011, 135, 234509-234520.

16. P. H. Hünenberger and M. Reif, Single-Ion Solvation, RSC Publishing, Cambridge UK, 2011.

17. M. B. Gee, N. R. Cox, Y. Jiao, N. Bentenitis, S. Weerasinghe and P. E. Smith, Journal of Chemical Theory and Computation, 2011, null-null.

18. J. Vincze, M. Valisko and D. Boda, The Journal of chemical physics, 2010, 133, 154507154506.

19. Y. V. Kalyuzhnyi, V. Vlachy and K. A. Dill, Physical Chemistry Chemical Physics, 2010, 12, 6260-6266.

20. I. Kalcher, J. C. F. Schulz and J. Dzubiella, Physical Review Letters, 2010, 104, 097802.

21. J. J. Howard, J. S. Perkyns and B. M. Pettitt, The journal of physical chemistry. B, 2010, 114, 6074-6083.

22. D. Fraenkel, The Journal of Physical Chemistry B, 2010, 115, 557-568.

23. D. Fraenkel, Molecular Physics, 2010, 108, 1435 - 1466.

24. L. Vrbka, M. Lund, I. Kalcher, J. Dzubiella, R. R. Netz and W. Kunz, The Journal of chemical physics, 2009, 131, 154109-154112.

25. Y. Luo and B. t. Roux, The Journal of Physical Chemistry Letters, 2009, 1, 183-189.

26. B. Li, SIAM Journal on Mathematical Analysis, 2009, 40, 2536-2566.

27. B. Li, Nonlinearity, 2009, 22, 811. 
28. W. Kunz and R. Neueder, in Specific Ion Effects, ed. W. Kunz, World Scientific Singapore, 2009, pp. 11-54.

29. W. Kunz, Specific Ion Effects, World Scientific Singapore, 2009.

30. Z. Abbas, E. Ahlberg and S. Nordholm, The Journal of Physical Chemistry B, 2009, 113, 5905-5916.

31. L. L. Lee, Molecular Thermodynamics of Electrolyte Solutions, World Scientific Singapore, 2008.

32. P. Jungwirth and B. Winter, Annual Review of Physical Chemistry, 2008, 59, 343-366.

33. P. Grochowski and J. Trylska, Biopolymers, 2008, 89, 93-113.

34. A. Grattoni, M. Merlo and M. Ferrari, The Journal of Physical Chemistry B, 2007, 111, 11770-11775.

35. W. R. Fawcett, Liquids, Solutions, and Interfaces: From Classical Macroscopic Descriptions to Modern Microscopic Details, Oxford University Press, New York, 2004.

36. K. J. Laidler, J. H. Meiser and B. C. Sanctuary, Physical Chemistry, Fourth edn., BrooksCole, Belmont CA, 2003.

37. T. N. Heinz, W. F. van Gunsteren and P. H. Hunenberger, The Journal of chemical physics, 2001, 115, 1125-1136.

38. K. S. Pitzer, Thermodynamics, 3rd edn., McGraw Hill, New York, 1995.

39. A. Chhih, O. Bernard, J. M. G. Barthel and L. Blum, Ber. Bunsenges. Phys. Chem., 1994, 98, 1516-1525.

40. V. S. Patwardhan and A. Kumar, AIChE Journal, 1993, 39, 711-714.

41. H. Cabezas and J. P. O'Connell, Industrial \& Engineering Chemistry Research, 1993, 32, 2892-2904.

42. K. S. Pitzer, Activity Coefficients in Electrolyte Solutions, CRC Press, Boca Raton FL USA, 1991.

43. J. F. Zemaitis, Jr., D. M. Clark, M. Rafal and N. C. Scrivner, Handbook of Aqueous Electrolyte Thermodynamics, Design Institute for Physical Property Data, American Institute of Chemical Engineers, New York, 1986.

44. J.-C. Justice, in Comprehensive Treatise of Electrochemistry Volume 5 Thermondynbamic and Transport Properties of Aqueous and Molten Electrolytes, eds. B. E. Conway, J. O. M. Bockris and E. Yaeger, Plenum, New York, 1983, pp. 223-338.

45. R. M. Pytkowicz, Activity Coefficients in Electrolyte Solutions, CRC, Boca Raton FL USA, 1979.

46. R. A. Robinson and R. H. Stokes, Electrolyte Solutions, Second edn., Butterworths Scientific Publications, London, 1959.

47. H. S. Harned and B. B. Owen, The Physical Chemistry of Electrolytic Solutions, Third edn., Reinhold Publishing Corporation, New York, 1958.

48. E. J. Maginn, AIChE Journal, 2009, 55, 1304-1310.

49. D. E. Post and L. G. Votta, Physics Today, 2005, 58, 35-41.

50. B. Eisenberg, Physics Today, 2005, 58, p.13 (Letter to the Editor).

51. O. Zikanov, Essential Computational Fluid Dynamics, Wiley, New York, 2010.

52. J. Anderson, Computational Fluid Dynamics, McGraw-Hill Science/Engineering/Math, 1995.

53. H. W. Engl, M. Hanke and A. Neubauer, Regularization of Inverse Problems Kluwer, Dordrecht, The Netherlands, 2000. 
54. J. Kaipio and E. Somersalo, Statistical and Computational Inverse Problems Springer, New York, 2005.

55. M. Burger, R. S. Eisenberg and H. Engl, SIAM J Applied Math, 2007, 67, 960-989

56. D. Boda, W. Nonner, D. Henderson, B. Eisenberg and D. Gillespie, Biophys. J., 2008, 94, 3486-3496.

57. D. Boda, M. Valisko, D. Henderson, B. Eisenberg, D. Gillespie and W. Nonner, J. Gen. Physiol., 2009, 133, 497-509.

58. D. Boda, W. Nonner, M. Valisko, D. Henderson, B. Eisenberg and D. Gillespie, Biophys. J., 2007, 93, 1960-1980.

59. D. Gillespie, Biophys J, 2008, 94, 1169-1184.

60. M. Lund, B. Jagoda-Cwiklik, C. E. Woodward, R. Vacha and P. Jungwirth, Journal Physical Chemistry Letters, 2010, 1, 300-303.

61. G. M. Kontogeorgis and G. K. Folas, Thermodynamic Models for Industrial Applications: From Classical and Advanced Mixing Rules to Association Theories, John Wiley \& Sons, Ltd, 2009.

62. C. A. Kraus, Bull. Amer. Math. Soc., 1938, 44, 361-383.

63. H. L. Friedman, Ionic Solution Theory, Interscience Publishers, New York, 1962.

64. P. M. V. Resibois, Electrolyte Theory, Harper \& Row, New York, 1968.

65. B. E. Conway, Electrochemical Data, Greenwood Press Publishers, Westport CT USA, 1969.

66. K. S. Pitzer and J. J. Kim, Journal of the American Chemical Society, 1974, 96, 57015707.

67. H. L. Friedman and W. D. T. Dale, in Statistical Mechanics, Part A: Equilibrium Techniques, ed. B. J. Berne, Plenum Press, Nwe York, 1977, vol. 1, pp. 85-135: Ch. 133.

68. A. L. Hovarth, Handbook of aqueous electrolyte solutions: physical properties, estimation, and correlation methods, Ellis Horwood,, New York, 1985.

69. V. S. Patwardhan and A. Kumar, AIChE Journal, 1986, 32, 1429-1438.

70. J. V. Sengers, R. F. Kayser, C. J. Peters and H. J. White, Jr., Equations of State for Fluids and Fluid Mixtures (Experimental Thermodynamics) Elsevier, New York, 2000.

71. R. Inchekel, J.-C. de Hemptinne and W. Fürst, Fluid Phase Equilibria, 2008, 271, 19-27.

72. R. T. Jacobsen, S. G. Penoncello, E. W. Lemmon and R. Span, in Equations of State for Fluids and Fluid Mixtures, eds. J. V. Sengers, R. F. Kayser, C. J. Peters and H. J. White, Jr., Elsevier, New York, 2000, pp. 849-882.

73. B. Larsen, Journal of Chemical Physics, 1976, 65, 3431-3438.

74. Y. Lin, K. Thomen and J.-C. d. Hemptinne, American Institute of Chemical Engineers AICHE Journal, 2007, 53, 989-1005.

75. N. Metropolis, A. W. Rosenbluth, M. N. Rosenbluth, A. H. Teller and E. Teller, The Journal of chemical physics, 1953, 21, 1087-1092.

76. J. A. Myers, S. I. Sandler and R. H. Wood, Industrial and Engineering Chemical Research, 2002, 41.

77. G. Odriozola and L. Berthier, The Journal of chemical physics, 2011, 134, 054504054509.

78. Y. Rosenfeld, Physical Review. E. Statistical Physics, Plasmas, Fluids, and Related Interdisciplinary Topics, 1996, 54, 2827-2838.

79. A. Kumar and V. S. Patwardhan, AIChE Journal, 1992, 38, 793-796. 
80. M. A. Kastenholz and P. H. Hunenberger, The Journal of chemical physics, 2006, 124, 224501-224520.

81. M. A. Kastenholz and P. H. Hunenberger, The Journal of chemical physics, 2006, 124, 124108-124112.

82. M. A. Kastenholz and P. H. Hunenberger, The Journal of chemical physics, 2006, 124, 124106-124127.

83. P. B. Petersen and R. J. Saykally, Annual Review of Physical Chemistry, 2006, 57, 333364.

84. H. Kokubo, J. Rosgen, D. W. Bolen and B. M. Pettitt, Biophys. J., 2007, 93, 3392-3407.

85. A. A. Kornyshev, J. Phys. Chem. B, 2007, 111, 5545-5557.

86. D. Boda and D. Henderson, Molecular Physics, 2008, 106, 2367-2370.

87. J. Che, J. Dzubiella, B. Li and J. A. McCammon, The journal of physical chemistry, 2008, 112, 3058-3069.

88. M. V. Fedorov and A. A. Kornyshev, The Journal of Physical Chemistry B, 2008, 112, 11868-11872.

89. I. S. Joung and T. E. Cheatham, The Journal of Physical Chemistry B, 2008, 112, 90209041.

90. I. V. Khavrutskii, J. Dzubiella and J. A. McCammon, The Journal of chemical physics, 2008, 128, 044106.

91. B.-Y. Dan, D. Andelman, D. Harries and R. Podgornik, Journal of Physics: Condensed Matter, 2009, 21, 424106.

92. D. Henderson and D. Boda, Physical Chemistry Chemical Physics, 2009, 11, 3822-3830.

93. D. Horinek, S. Mamatkulov and R. Netz, The Journal of chemical physics, 2009, 130, 124507-124521.

94. J. G. Ibarra-Armenta, A. Martin-Molina and M. Quesada-Perez, Physical Chemistry Chemical Physics, 2009, 11, 309-316.

95. J. JaneCek and R. R. Netz, The Journal of chemical physics, 2009, 130, 074502-074515.

96. I. Kalcher and J. Dzubiella, The Journal of chemical physics, 2009, 130, 134507.

97. S. Buyukdagli, M. Manghi and J. Palmeri, Physical Review Letters, 2010, 105, 158103.

98. I. Kalcher, J. C. F. Schulz and J. Dzubiella, The Journal of chemical physics, 2010, 133, 164511-164515.

99. F. Lipparini, G. Scalmani, B. Mennucci, E. Cances, M. Caricato and M. J. Frisch, The Journal of chemical physics, 2010, 133, 014106-014111.

100. H. Paul and S. Matthias, Journal of Physics: Condensed Matter, 2010, 22, 325108.

101. G. b. Rutkai, D. Boda and T. s. Kristóf, The Journal of Physical Chemistry Letters, 2010, 1, 2179-2184.

102. H. Saidani, N. Ben Amar, J. Palmeri and A. Deratani, Langmuir : the ACS journal of surfaces and colloids, 2010, 26, 2574-2583.

103. J. Sala, E. Guardia and J. Marti, The Journal of chemical physics, 2010, 132, 214505214511.

104. H. Yu, T. W. Whitfield, E. Harder, G. Lamoureux, I. Vorobyov, V. M. Anisimov, A. D. MacKerell and B. t. Roux, Journal of Chemical Theory and Computation, 2010, 6, 774786.

105. C. Zhang, S. Raugei, B. Eisenberg and P. Carloni, Journal of Chemical Theory and Computation, 2010, 6, 2167-2175. 
106. B. Li, X. Cheng and Z. Zhang, SIAM Journal on Applied Mathematics, 2011, 71, 20932111.

107. S. Zhao, Z. Jin and J. Wu, The Journal of Physical Chemistry B, 2011, null-null.

108. N. Schwierz, D. Horinek and R. R. Netz, Langmuir, 2010, 26, 7370-7379.

109. B. Eisenberg, Chemical Physics Letters, 2011, 511, 1-6.

110. B. Eisenberg, Posted on arXiv.org with Paper ID arXiv:1105.0184v1, 2011.

111. B. Eisenberg, in Advances in Chemical Physics, John Wiley \& Sons, Inc., 2011, pp. 77223 also available at http: \larix.org as arXiv 1009.1786v1001

112. B. Eisenberg, Y. Hyon and C. Liu, Journal of Chemical Physics, 2010, 133, 104104

113. Y. Hyon, B. Eisenberg and C. Liu, Communications in Mathematical Sciences, 2011, 9 , 459-475 also available as preprint 2318 (IMA, University of Minnesota, Minneapolis) http://www.ima.umn.edu/preprints/jun2010/jun2010.html, 2010.

114. Y. Hyon, J. E. Fonseca, B. Eisenberg and C. Liu, Biophysical Journal, 2011, 100, 578a.

115. Y. Mori, C. Liu and R. S. Eisenberg, Physica D: Nonlinear Phonomena 2011, 240, 18351852.

116. A. Singer, Z. Schuss and R. S. Eisenberg, Journal of Statistical Physics, 2005, 119, 1397-1418.

117. Q. Zheng and G.-W. Wei, The Journal of chemical physics, 2011, 134, 194101-194117.

118. Y. Hyon, D. Y. Kwak and C. Liu, available at URL: http://www.ima.umn.edu as IMA Preprint Series \# 2228, 2010, 26, 1291 - 1304, available at URL: http://www.ima.umn.edu as IMA Preprint Series \# 2228.

119. Y. Hyon, Q. Du and C. Liu, Journal of Computational and Theoretical Nanoscience, 2010, 7, 756-765.

120. C. Liu, in Multi-scale Phenomena in Complex Fluids: Modeling, Analysis and Numerical Simulations, ed. T. Y. Hou, Liu, C., Liu, J.-g, World Scientific Publishing Company, Singapore, 2009.

121. M. Doi, Journal of the Physical Society of Japan, 2009, 78, 052001.

122. J. Zhang, X. Gong, C. Liu, W. Wen and P. Sheng, Physical Review Letters, 2008, 101, 194503.

123. P. Sheng, J. Zhang and C. Liu, Progress of Theoretical Physics Supplement No. 175, 2008, 131-143.

124. Y. Hyon, J. A. Carrillo, Q. Du and C. Liu, Kinetic and Related Models, 2008, 1, 171-184.

125. F.-H. Lin, C. Liu and P. Zhang, Communications on Pure and Applied Mathematics, 2007, 60, 838-866.

126. F.-H. Lin, C. Liu and P. Zhang, Communications on Pure and Applied Mathematics, 2005, 58, 1437-1471.

127. R. G. Larson, The Structure and Rheology of Complex Fluids Oxford, New York, 1995.

128. P.-G. d. Gennes and J. Prost, The Physics of Liquid Crystals, Oxford University Press, New York, 1993.

129. M. Doi and S. F. Edwards, The Theory of Polymer Dynamics, Oxford University Press, New York, 1988.

130. L. Rayleigh, previously John Strutt, Proceedings of the London Mathematical Society, 1873, IV, 357-368.

131. L. Rayleigh, previously John Strutt, Phil. Mag., 1892, 33, 209.

132. L. Rayleigh, previously John Strutt, Theory of Sound, 2 edn., Dover Reprint 1976, New York, 1896. 
133. S. Machlup and L. Onsager, Physical Review, 1953, 91, 1512.

134. L. Onsager, Physical Review, 1931, 38, 2265-2279.

135. L. Onsager, Physical Review, 1931, 37, 405-109.

136. L. Onsager and S. Machlup, Phys. Rev, 1953, 91:, 1505-1512.

137. M. Doi, Journal of Physics of Condensed Matter, 2011, 23, 284118.

138. M. A. Biot, Variational Principles in Heat Transfer: A Unified Lagrangian Analysis of Dissipative Phenomena, Oxford University Press, New York, 1970.

139. E. Sciubba, International Journal of Thermodynamics, 2004, 7, 115-122.

140. T. Y. Hou, C. Liu and J.-g. Liu, Multi-scale Phenomena in Complex Fluids: Modeling, Analysis and Numerical Simulations, World Scientific Publishing Company, Singapore, 2009.

141. S. Buyukdagli, M. Manghi and J. Palmeri, Physical Review E, 2010, 81, 041601.

142. G.-W. Wei, Bulletin of Mathematical Biology, 2010, 72, 1562-1622.

143. R. J. Goldston and P. H. Rutherford, Introduction to Plasma Physics, Institute of Physics Publishing, London, 1995.

144. R. M. Kulsrud, Plasma Physics for Astrophysics, Princeton, Princeton, NJ, USA, 2005.

145. E. Mason and E. McDaniel, Transport Properties of Ions in Gases, John Wiley and Sons, NY, 1988.

146. W. Van Roosbroeck, Bell System Technical Journal, 1950, 29, 560-607.

147. J. R. Macdonald, Physical Review, 1953, 92, 4-17.

148. P. A. Markowich, C. A. Ringhofer and C. Schmeiser, Semiconductor Equations, Springer-Verlag, New York, 1990.

149. J. W. Jerome, Analysis of Charge Transport. Mathematical Theory and Approximation of Semiconductor Models, Springer-Verlag, New York, 1995.

150. M. Lundstrom, Fundamentals of Carrier Transport, Second Edition edn., AddisonWesley, NY, 2000.

151. S. Selberherr, Analysis and Simulation of Semiconductor Devices, Springer-Verlag, New York, 1984.

152. R. Eisenberg and D. Chen, Biophysical Journal, 1993, 64, A22.

153. R. de Levie and H. Moreira, Journal of Membrane Biology, 1972, 9, 241-260.

154. D. G. Levitt, Biophys J, 1975, 15, 533-551.

155. D. G. Levitt, Biophys. J, 1982, 37, 575-587.

156. K. Cooper, E. Jakobsson and P. Wolynes, Progress in Biophysics and Molecular Biology, 1985, 46, 51-96.

157. D. G. Levitt, Biophys. J., 1985, 52, 575-587.

158. I. Rubinstein, Electro-diffusion of ions, SIAM, Philadelphia, 1990.

159. V. Barcilon, D. P. Chen and R. S. Eisenberg, Society of Industrial and Applied Mathematics Journal of Applied Mathematics, 1992, 52, 1405-1425.

160. D. Chen, V. Barcilon and R. S. Eisenberg, Biophys. J, 1992, 61, A11.

161. D. P. Chen, V. Barcilon and R. S. Eisenberg, Biophys J, 1992, 61, 1372-1393.

162. D. P. Chen and R. S. Eisenberg, Biophys. J, 1993, 64, 1405-1421.

163. R. S. Eisenberg, J. Membrane Biol., 1996, 150, 1-25. Also available on http:Ilarxiv.org as arXiv 1009.2857.

164. R. S. Eisenberg, in New Developments and Theoretical Studies of Proteins, ed. R. Elber, World Scientific, Philadelphia, 1996, vol. 7, pp. 269-357. Published in the Physics ArXiv as arXiv:0807.0715. 
165. V. Barcilon, D.-P. Chen, R. S. Eisenberg and J. W. Jerome, SIAM J. Appl. Math., 1997, 57, 631-648.

166. D. P. Chen, J. Lear and R. S. Eisenberg, Biophys. J., 1997, 72, 97-116.

167. S.-H. Chung, M. Hoyles, T. Allen and S. Kuyucak, Biophysical Journal, 1998, 75, 793809.

168. W. Nonner and B. Eisenberg, Biophys. J., 1998, 75, 1287-1305.

169. B. Corry, S. Kuyucak and S. H. Chung, J Gen Physiol, 1999, 114, 597-599.

170. G. R. Dieckmann, J. D. Lear, Q. Zhong, M. L. Klein, W. F. DeGrado and K. A. Sharp, Biophysical Journal, 1999, 76, 618-630.

171. R. S. Eisenberg, Journal of Membrane Biology, 1999, 171, 1-24.

172. D. Gillespie, Rush, 1999.

173. U. Hollerbach, D. Chen, W. Nonner and B. Eisenberg, Biophysical Journal, 1999, 76, A205.

174. M. G. Kurnikova, R. D. Coalson, P. Graf and A. Nitzan, Biophysical Journal, 1999, 76, 642-656.

175. A. E. Cardenas, R. D. Coalson and M. G. Kurnikova, Biophysical Journal, $2000,79$.

176. B. Corry, S. Kuyucak and S. H. Chung, Biophys J, 2000, 78, 2364-2381.

177. B. Eisenberg, in Biophysics Textbook On Line "Channels, Receptors, and Transporters" http://www.biophysics.org/btol/channel.html\#5, ed. L. J. DeFelice, Published in ArXiv as arXiv:0807.0721, 2000.

178. P. Graf, A. Nitzan, M. G. Kurnikova and R. D. Coalson, Journal of Physical Chemistry $B, 2000,104,12324-12338$.

179. U. Hollerbach, D. P. Chen, D. D. Busath and B. Eisenberg, Langmuir, 2000, 16, 55095514.

180. W. Im, S. Seefeld and B. Roux, Biophysical Journal, 2000, 79, 788-801.

181. G. Moy, B. Corry, S. Kuyucak and S. H. Chung, Biophys J, 2000, 78, 2349-2363.

182. W. Im and B. Roux, Biophysical Journal, 2001, 115, 4850-4861.

183. Z. Schuss, B. Nadler and R. S. Eisenberg, Phys Rev E Stat Nonlin Soft Matter Phys, 2001, 64, 036116.

184. T. A. van der Straaten, R. S. Eisenberg, J. M. Tang, U. Ravaioli and N. Aluru, Biophysical Journal, 2001, 80, 115a.

185. D. Gillespie, W. Nonner and R. S. Eisenberg, Journal of Physics (Condensed Matter), 2002, 14, 12129-12145.

186. U. Hollerbach, D.-P. Chen and R. S. Eisenberg, Journal of Computational Science, 2002, 16, 373-409.

187. U. Hollerbach and R. Eisenberg, Langmuir, 2002, 18, 3262-3631.

188. W. Im and B. Roux, Journal of molecular biology, 2002, 322, 851-869.

189. W. Im and B. Roux, Journal of molecular biology, 2002, 319, 1177-1197.

190. Z. Schuss, B. Nadler, A. Singer and R. Eisenberg, AIP Conference Proceedings , 3-6 September 2002: Unsolved Problems Of Noise And Fluctuations, UPoN 2002, 3rd International Conference on Unsolved Problems of Noise and Fluctuations in Physics, Biology, and High Technology Washington, DC, 2002.

191. B. Corry, S. Kuyucak and S. H. Chung, Biophys J, 2003, 84, 3594-3606.

192. B. Eisenberg, Journal of Computational Electronics, 2003, 2, 245-249.

193. A. B. Mamonov, R. D. Coalson, A. Nitzan and M. G. Kurnikova, Biophys J, 2003, 84, 3646-3661. 
194. B. Nadler, U. Hollerbach and R. S. Eisenberg, Phys Rev E Stat Nonlin Soft Matter Phys, 2003, 68, 021905.

195. T. A. van der Straaten, J. M. Tang, U. Ravaioli, R. S. Eisenberg and N. R. Aluru, Journal of Computational Electronics, 2003, 2, 29-47.

196. M. Aguilella-Arzo, V. Aguilella and R. S. Eisenberg, European Biophysics Journal,, 2005, 34, 314-322.

197. R. D. Coalson and M. G. Kurnikova, IEEE transactions on nanobioscience, 2005, 4, 8193.

198. B. Corry and S.-H. Chung, European Biophysics Journal, 2005, 34, 208-216.

199. B. Lu, D. Zhang and J. A. McCammon, The Journal of chemical physics, 2005, 122, $214102-214107$.

200. M. Saraniti, S. Aboud and R. Eisenberg, Reviews in Computational Chemistry, 2006, 22, 229-294.

201. B. Eisenberg and W. Liu, SIAM Journal on Mathematical Analysis 2007, 38, 1932-1966.

202. N. Abaid, R. S. Eisenberg and W. Liu, SIAM Journal of Applied Dynamical Systems, 2008, 7, 1507.

203. A. Singer, D. Gillespie, J. Norbury and R. S. Eisenberg, European Journal of Applied Mathematics, 2008, 19, 541-560.

204. K. Arning, Johannes Kepler University Linz, 2009.

205. D. G. Luchinsky, R. Tindjong, I. Kaufman, P. V. E. McClintock and R. S. Eisenberg, Physical Review E (Statistical, Nonlinear, and Soft Matter Physics), 2009, 80, 021925021912.

206. A. Singer and J. Norbury, SIAM J Appl Math, 2009, 70, 949-968.

207. W. Liu and B. Wang, J. Dynam. Differential Equations, 2010, 22, 413-437.

208. C. Hsiao-Bing, C. Li-Tien and L. Bo, Nonlinearity, 2011, 24, 3215.

209. S. Zhou, Z. Wang and B. Li, Physical Review E 2011, 84.

210. E. Csanyi, D. Boda, D. Gillespie and T. Kristof, Biochimica et Biophysica Acta, 2012, 1818, 592-600.

211. B. Eisenberg, Fluctuations and Noise Letters, 2012, (in the press). .

212. R. Tindjong, I. Kaufman, P. V. E. Mcclintock, D. G. Luchinsky and R. S. Eisenberg, Fluctuation and Noise Letters, 2012, 11, 83-93.

213. M. Z. Bazant, M. S. Kilic, B. D. Storey and A. Ajdari, Advances in Colloid and Interface Science, 2009, 152, 48-88.

214. M. Z. Bazant, K. Thornton and A. Ajdari, Physical Review E, 2004, 70, 021506.

215. B. Richard P, Journal of Membrane Science, 1984, 17, 1-62.

216. K. Arning, M. Burger, R. S. Eisenberg, H. W. Engl and L. He, PAMM, 2007, 7, $1120801-$ 1120802.

217. D. G. Levitt, $J$ Gen Physiol, 1999, 113, 789-794.

218. A. B. Mamonov, M. G. Kurnikova and R. D. Coalson, Biophysical chemistry, 2006, 124, 268-278.

219. Damocles, in http://www.research.ibm.com/DAMOCLES/home.html, 2007.

220. W. Shockley, Electrons and Holes in Semiconductors to applications in transistor electronics, van Nostrand, New York, 1950.

221. B. G. Streetman, Solid State Electronic Devices, 4th edn., Prentice Hall, Englewood Cliffs, NJ, 1972.

222. S. M. Sze, Physics of Semiconductor Devices, John Wiley \& Sons, New York, 1981. 
223. D. J. Rouston, Bipolar Semiconductor Devices, McGraw-Hill Publishing Company,, New York, 1990.

224. M. Shur, Physics of Semiconductor Devices, Prentice Hall, New York, 1990.

225. R. F. Pierret, Semiconductor Device Fundamentals, Addison Wesley, New York, 1996.

226. R. T. Howe and C. G. Sodini, Microelectronics: an integrated approach, Prentice Hall, Upper Saddle River, NJ USA, 1997.

227. D. L. Critchlow, Proceedings of the IEEE, 1999, 87, 659-667.

228. P. R. Gray, P. J. Hurst, S. H. Lewis and R. G. Meyer, Analysis and Design of Analog Integrated Circuits, 4th Edition edn., John Wiley, New York, 2001.

229. S. J. Fiedziuszko, I. C. Hunter, T. Itoh, Y. Kobayashi, T. Nishikawa, S. Stitzer and K. Wakino, IEEE Transactions on Microwave Theory and Techniques, 2002, 50, 706-720.

230. J. D. Cressler, Silicon Heterostructure Handbook: Materials, Fabrication, Devices, Circuits and Applications of SiGe and Si Strained-Layer Epitaxy, CRC Boca Raton, FL, 2005.

231. K. Varsos, J. Luntz, M. Welsh and K. Sarabandi, Proceedings of the IEEE, 2011, 99, 2112-2124.

232. A. Ben-Naim, Molecular Theory of Water and Aqueous Solutions Part II: The Role of Water in Protein Folding, Self-Assembly and Molecular Recognition World Scientific Publishing Company, 2011.

233. R. M. Fuoss and C. A. Kraus, Journal of the American Chemical Society, 1933, 55, 10191028.

234. R. M. Fuoss and C. A. Kraus, Journal of the American Chemical Society, 1933, 55, 23872399.

235. R. M. Fuoss, Chemical Reviews, 1935, 17, 27-42.

236. R. M. Fuoss and L. Onsager, Proceedings of the National Academy of Sciences of the United States of America, 1955, 41, 274-283.

237. R. M. Fuoss and L. Onsager, The journal of physical chemistry, 1958, 62, 1339-1340.

238. R. M. Fuoss, The journal of physical chemistry, 1959, 63, 633-636.

239. R. M. Fuoss and F. Accascina, Electrolytic Conductance, Interscience, New York, 1959.

240. R. M. Fuoss and L. Onsager, The journal of physical chemistry, 1962, 66, 1722-1726.

241. R. M. Fuoss and L. Onsager, The journal of physical chemistry, 1962, 66, 1722-1726.

242. R. M. Fuoss, Journal of the American Chemical Society, 1963, 85, 2536-2536.

243. R. M. Fuoss and L. Onsager, The journal of physical chemistry, 1963, 67, 621-628.

244. R. M. Fuoss, The journal of physical chemistry, 1974, 78, 1383-1386.

245. R. M. Fuoss, The journal of physical chemistry, 1975, 79, 525-540.

246. J. E. Anderson, Journal of Non-Crystalline Solids, 1994, 172-174, Part 2, 1190-1194.

247. S. Durand-Vidal, P. Turq, O. Bernard, C. Treiner and L. Blum, Physica A, 1996, 231, 123-143.

248. S. Van Damme and J. Deconinck, The Journal of Physical Chemistry B, 2007, 111, 53085315.

249. G. M. Roger, S. Durand-Vidal, O. Bernard and P. Turq, The journal of physical chemistry. B, 2009, 113, 8670-8674.

250. S. Van Damme, N. Smets, D. De Wilde, G. Weyns and J. Deconinck, The Journal of Physical Chemistry B, 2009, 113, 3105-3111.

251. S. Van Damme, N. Smets, D. De Wilde, G. Weyns and J. Deconinck, The Journal of Physical Chemistry A, 2009, 113, 4972-4975. 
252. T. Yamaguchi, T. Matsuoka and S. Koda, The Journal of chemical physics, 2011, 135, 164511-164510.

253. D. Boda and D. Gillespie, Journal of Chemical Theory and Computation, 2012, 8, 824829.

254. D. Chen, R. Eisenberg, J. Jerome and C. Shu, Biophysical J., 1995, 69, 2304-2322.

255. S. Hagiwara and S. Nakajima, J Gen Physiol, 1966, 49, 793-806.

256. P. G. Kostyuk, O. A. Krishtal and P. A. Doroshenko, Pflugers Arch, 1974, 348, 83-93.

257. S. Hagiwara and L. Byerly, Annual review of neuroscience, 1981, 4, 69-125.

258. W. Almers and E. W. McCleskey, J.Physiol., 1984, 353, 585-608.

259. P. Hess and R. W. Tsien, Nature, 1984, 309, 453-456.

260. E. W. McCleskey and W. Almers, Proceedings of the National Academy of Science USA, 1985, 82, 7149-7153.

261. S. H. Heinemann, H. Terlau, W. Stuhmer, K. Imoto and S. Numa, Nature, 1992, 356, 441-443.

262. E. W. McCleskey, M. D. Womack and L. A. Fieber, International review of cytology, 1993, 137C, 39-54.

263. P. T. Ellinor, J. Yang, W. A. Sather, J.-F. Zhang and R. Tsien, Neuron, 1995, 15, 11211132.

264. E. W. McCleskey, Biophys J, 2000, 79, 1691-1692.

265. W. A. Sather and E. W. McCleskey, Annual review of physiology, 2003, 65, 133-159.

266. B. Eisenberg, Institute of Mathematics and its Applications, 2009, IMA University of Minnesota http://www.ima.umn.edu/2008-2009/W2012.2008-2012.2008/abstracts.html and also http://arxiv.org/0906.5173.

267. D. Boda, J. Giri, D. Henderson, B. Eisenberg and D. Gillespie, The Journal of chemical physics, 2011, 134, 055102-055114.

268. D. Krauss, B. Eisenberg and D. Gillespie, European Biophysics Journal, 2011, 40, $775-$ 782.

269. A. L. Hodgkin, Biological Reviews, 1951, 26, 339-409.

270. A. L. Hodgkin and A. F. Huxley, J. Physiol., 1952, 116, 449-472.

271. I. Atwater, F. Bezanilla and E. Rojas, J Physiol, 1969, 201, 657-664.

272. F. Bezanilla, J. Vergara and R. E. Taylor, in Methods of Experimental Physics Volume 20, eds. G. Ehrenstein and H. Lecar, Elsevier Inc., New York, 1982, vol. 20, pp. 445511.

273. F. Bezanilla, The Journal of membrane biology, 1985, 88, 97-111.

274. F. Bezanilla, Biophys J, 1987, 52, 1087-1090.

275. A. M. Correa, R. Latorre and F. Bezanilla, J Gen Physiol, 1991, 97, 605-625.

276. F. Bezanilla, Neuron, 2008, 60, 456-468.

277. J. Payandeh, T. Scheuer, N. Zheng and W. A. Catterall, Nature, 2011, 475, 353-358.

278. J. Giri, J. E. Fonseca, D. Boda, D. Henderson and B. Eisenberg, Phys Biol, 2011, 8, 026004.

279. B. Eisenberg, Biophysical chemistry, 2003, 100, 507 - 517.

280. B. Hille, Ionic Channels of Excitable Membranes, 3rd edn., Sinauer Associates Inc., Sunderland, 2001.

281. J. R. Pappenheimer, Physiol. Rev., 1953, 33, 387-423.

282. J. R. Pappenheimer, Annual review of physiology, 1987, 49, 1-16. 
283. Y. Hyon, J. E. Fonseca, B. Eisenberg and C. Liu, Discrete and Continuous Dynamical Systems - Series B (DCDS-B) in the press, 2012.

284. D. Boda, K.-Y. Chan and D. Henderson, The Journal of chemical physics, 1998, 109, $7362-7371$.

285. S. Varga, D. Boda, D. Henderson and S. Sokolowski, J Colloid Interface Sci, 2000, 227, 223-226.

286. J. Reszko-Zygmunt, S. Sokolowski, D. Henderson and D. Boda, The Journal of chemical physics, 2005, 122, 84504 .

287. D. Boda, K. Y. Chan and I. Szalai, Molecular Physics, 1997, 92, 1067-1072.

288. A. Malasics, D. Gillespie and D. Boda, Journal of Chemical Physics, 2008, 128, 124102.

289. J. Vincze, M. Valisko and D. Boda, The Journal of chemical physics, 2010, 133, 154507.

290. A. S. Fairhurst and D. J. Jenden, Proceedings of the National Academy of Sciences of the United States of America, 1962, 48, 807-813.

291. D. J. Jenden and A. S. Fairhurst, Pharmacol Rev, 1969, 21, 1-25.

292. G. Meissner, The Journal of biological chemistry, 1986, 261, 6300-6306.

293. M. Fill and J. A. Copello, Physiological reviews, 2002, 82, 893-922.

294. G. Meissner, Front Biosci, 2002, 7, d2072-2080.

295. D. Gillespie, L. Xu, Y. Wang and G. Meissner, Journal of Physical Chemistry, 2005, 109, 15598-15610.

296. Y. Wang, L. Xu, D. Pasek, D. Gillespie and G. Meissner, Biophysical Journal, 2005, 89, 256-265.

297. T. Nagrani, M. Siyamwala, G. Vahid and S. Bekheit, Neurologist, 2011, 17, 91-94.

298. D. Gillespie, W. Nonner and R. S. Eisenberg, Physical Review E, 2003, 68, 0313503.

299. D. Gillespie, M. Valisko and D. Boda, Journal of Physics: Condensed Matter 2005, 17, 6609-6626.

300. D. Gillespie and D. Boda, Biophys. J., 2008, 95, 2658-2672.

301. D. Gillespie and M. Fill, Biophys. J., 2008, 95, 3706-3714.

302. D. Gillespie, J. Giri and M. Fill, Biophyiscal Journal, 2009, 97, pp. 2212 - 2221

303. D. Krauss and D. Gillespie, European Biophysics Journal, 2010, 39, 1513-1521.

304. R. M. Fuoss and L. Onsager, Proceedings of the National Academy of Sciences of the United States of America, 1961, 47, 818-825.

305. J. Barthel, H. Krienke and W. Kunz, Physical Chemistry of Electrolyte Solutions: Modern Aspects, Springer, New York, 1998.

306. B. Eisenberg, Fluctuations and Noise Letters, 2012, 11, 76-96 Earlier version 'Living Transistors: a Physicist's View of Ion Channels' available on http://arxiv.org/ as qbio/0506016v0506012.

307. S. Aboud, D. Marreiro, M. Saraniti and R. Eisenberg, J. Computational Electronics, 2004, 3, 117-133.

308. J. R. Barker and D. K. Ferry, Solid-State Electronics, 1980, 23, 531-544.

309. D. K. Ferry, Semiconductor Transport, Taylor and Francis, New York, 2000.

310. M. Goryll, S. Wilk, G. M. Laws, T. J. Thornton, S. M. Goodnick, M. Saraniti, J. M. Tang and R. S. Eisenberg, Superlattices \& Microstructures, 2003, 34, 451-457.

311. K. Hess, Monte Carlo Device Simulation: Full Band and Beyond, Kluwer, Boston, MA USA, 1991.

312. K. Hess, Advanced Theory of Semiconductor Devices, IEEE Press, New York, 2000. 
313. K. Hess, J. P. Leburton and U. Ravaioli, Computational Electronics: Semiconductor Transport and Device Simulation, Kluwer, Boston, MA USA, 1991.

314. M. Saraniti, S. Hu, S. Goodnick and S. J. Wigger, Physica B, 2002, in the press.

315. M. Saraniti, S. J. Wigger, Z. Schuss and R. S. Eisenberg, MSM (Microsystems), 2002, 5, in the press.

316. T. A. van der Straaten, G. Kathawala, R. S. Eisenberg and U. Ravaioli, Molecular Simulation, 2004, 31, 151-171.

317. T. A. van der Straaten, J. Tang, R. S. Eisenberg, U. Ravaioli and N. R. Aluru, J. Computational Electronics 2002, 1, 335-340.

318. K. E. Cooper, P. Y. Gates and R. S. Eisenberg, Quarterly Review of Biophysics, 1988, 21, 331-364.

319. R. S. Eisenberg, Journal of Membrane Biology, 1990, 115, 1-12. Available on arXiv as http://arxiv.org/abs/1112.2363.

320. D. Chen, L. Xu, A. Tripathy, G. Meissner and R. Eisenberg, Biophys. J., 1997, 73, 13491354.

321. B. Eisenberg, available on http://arxiv.org/ as q-bio/0506016v2 24 pages, 2005. 